\title{
Bare Foot and In-shoe Plantar Pressure in Diabetic Males and Females - Is There Difference?
}

\author{
Saleh S Altayyar \\ Biomedical Technology Department, \\ College of Applied Medical Sciences, King \\ Saud University, Riyadh, Saudi Arabia
}

Purpose: High plantar pressure is one of the factors associated with foot ulceration in diabetic patients. High-risk limbs could eventually be identified through this approach. The study was conducted to evaluate the difference in the barefoot and in-shoe plantar pressure among diabetic males and females.

Patients and Methods: A cross-sectional study was conducted and purposive sampling was employed for the recruitment of subjects in King Abdullah walking center. The dynamic plantar pressure generated by each subject was recorded using "novel footprint software" and up to five successful trials were collected for each subject of right and left foot.

Results: The mean age of female and male patients was $50.6 \pm 13.4$ and $46.07 \pm 11.17$, respectively. The mean difference between the weights was higher in males. The barefoot peak plantar pressure between gender in left limb was found significant. Moreover, the mean difference in plantar pressure at maximum concentration and maximum force of right and left limb between males and females was found statistically significant. The mean difference in in-shoe plantar pressure at maximum force of left limb between males and females was found statistically significant.

Conclusion: As the prevalence of diabetes is increasing, the risk of plantar pressure also increasing simultaneously. The difference in plantar pressure among diabetic males and females is critically important as our study indicated that the bare foot and in-shoe plantar pressure was found higher in males than females as males had higher weight than females. Further longitudinal studies are required to be conducted in this context.

Keywords: diabetes mellitus, foot injuries, insoles, neuropathy, plantar pressure

\section{Introduction}

The occurrence of diabetes mellitus (DM) due to paucity of insulin production or insulin incompetence is commonly found in affecting normal functions of a human body. ${ }^{1} \mathrm{DM}$ is considered the 7 th cause of death in the world. ${ }^{2}$ According to the International Diabetes Federation (IDF), there are 382 million people worldwide with diabetes in $2013 .{ }^{3}$ IDF expects that the number of people with diabetes will reach 592 million in 2035 ( 1 in every 10 people will have diabetes). ${ }^{3}$ The prevalence of diabetes among Saudi population aged 20-79 years is $23.9 \%{ }^{4,5}$ Saudi Arabia has the highest prevalence of diabetes compared to its neighboring Gulf countries. $^{4,5}$ Prevalence of diabetes among Saudi Arabia's population is among the highest around the world, with approximately 2 million people diagnosed with the disease by 2010 and 3.8 million in $2014 .^{6}$

Plantar pressure is an approach to measure the pressure between support surface and food on a regular basis for locomotor activities. It becomes important to derive
Correspondence: Saleh S Altayyar

Tel +966505456035

Email stayyar@ksu.edu.sa 
such data in gait and posture research to investigate sport biomechanics, injury prevention, footwear design, and lower limb issues. ${ }^{7}$ Footwear is very important for protecting the feet from excessive pressure and providing stability during walking and other daily activities. Footwear design is very significant for the health of the foot, characteristics of foot varies across race, gender, environment, lifestyle and, therefore, population. These variances should be considered when designing the arch insoles.

Biomechanical impairments are led by diabetic neuropathy, which results in impaired plantar foot pressure. ${ }^{8}$ Increased risks of foot ulcer among patients are due to neuropathy and foot deformity. ${ }^{8}$ The occurrence of mostly foot ulcers and injuries is because of clawed toes and metatarsal heads inclined to repetitive increased plantar pressure as well as shear stress. ${ }^{9}$ Foot supports $95 \%$ of the overall body weight and holds the ground force throughout gait. ${ }^{10,11}$ Impaired sensation in lower extremity is witnessed among $20-50 \%$ of diabetic patients, ${ }^{12}$ which consequently directs toward impaired weight bearing trend on the foot's plantar surface. ${ }^{13}$ Foot ulceration is commonly the most repeated cause of amputation among individuals with diabetes. ${ }^{14}$ More than $85 \%$ of diabetes is related with lower extremity amputation. ${ }^{15}$ Previous studies have reported the increase in dynamic foot pressure, which consequently leads toward the development of diabetic foot ulcer. ${ }^{16-23}$ The initial standard treatment protocol for diabetic feet encompasses of foot care and proper shoes. $^{2}$

Plantar insoles are usually utilized in treating musculoskeletal pain and issues in the lower limbs. In particular, scientific findings have reported the prescription of this device for deteriorating plantar pressure, enhancing foot ulcers caused by diabetes, and relieving pain in the lumbar region and lower limbs. ${ }^{24,25}$ The insole is one of the economical, essential, and practical approaches for reducing and preventing foot ulceration in diabetic patients. Venous blood flow, weight-bearing joints, and intervertebral stress were affected through prolonged standing, and thus, cause discomfort and pain as well as worsen musculoskeletal circumstances. ${ }^{26}$

There are few clinical trials that address the use of and response to insoles in the standing posture in spite of the evidence nature of the issue. Thereby, it is essential to evaluate the effect of insole wearing on the plantar pressure of diabetic patients. ${ }^{27}$ The use of insoles has been examined by few studies for verifying the reduction of complaints, regardless of relating them with weight- bearing modifications or other mechanical constructs that may demonstrate the modification. ${ }^{37}$ The effectiveness of this intervention has been assessed in few studies, but none have made comparisons between different insoles in a work environment. In this regard, these gaps in knowledge for interventions intended at static and standing positions should be addressed in symptomatic workers with respect to comparisons of insoles and plantar behaviour found on the market. ${ }^{28,29}$ Plantar pressure can be influenced by different material density and thickness of the orthopaedic insoles.

According to our extensive literature search, Mickle \& Steele's ${ }^{30}$ study was the only one to look at the impact of obesity on the plantar pressure distribution, and it found that obese people had considerably higher peak pressure. Obese feet were shown to have a flatter structure and more plantar soft tissue thickness. ${ }^{30}$ Different body distribution patterns have an impact on plantar pressure. Limited data is available in this regard, therefore we planned this study to observe the mean difference in plantar pressure at peak concentration, maximum concentration and maximum force between diabetic males and females.

\section{Patients and Methods}

A cross sectional study was conducted and purposive sampling was employed for the recruitment of subjects in King Abdullah walking center. A total of sixty subjects (30 male; 30 female) were selected. After removing the participant's shoes and socks, weight was measured by using mechanical scale and dynamic plantar pressure was measured by using a portable platform NOVEL AT - 4 (Figure 1).

Information related to persuaders was provided to subjects. Informants were asked to walk straight with head in front of wall regardless of targeting the pressure platform. To ensure repeatability, up to five successful trials were collected for each subject of right and left foot. The dynamic plantar pressure generated by each subject was recorded using "novel footprint software".

Subjects were given custom made insoles designed and manufactured for each subject using their plantar pressure profile, the insoles were used with appropriate shoes with a Velcro ${ }^{\circledR}$ Dacron-backed closure or laces to fasten the shoe. The first metatarsophalangeal joint was lodged in the widest aspect of the shoe and 1 to $1.25 \mathrm{~cm}$ between the end of shoe and the longest toe was allowed.

Data collection commenced with demographic and anthropometric data and information on duration of the diabetic mellitus. A single examiner has performed all the 


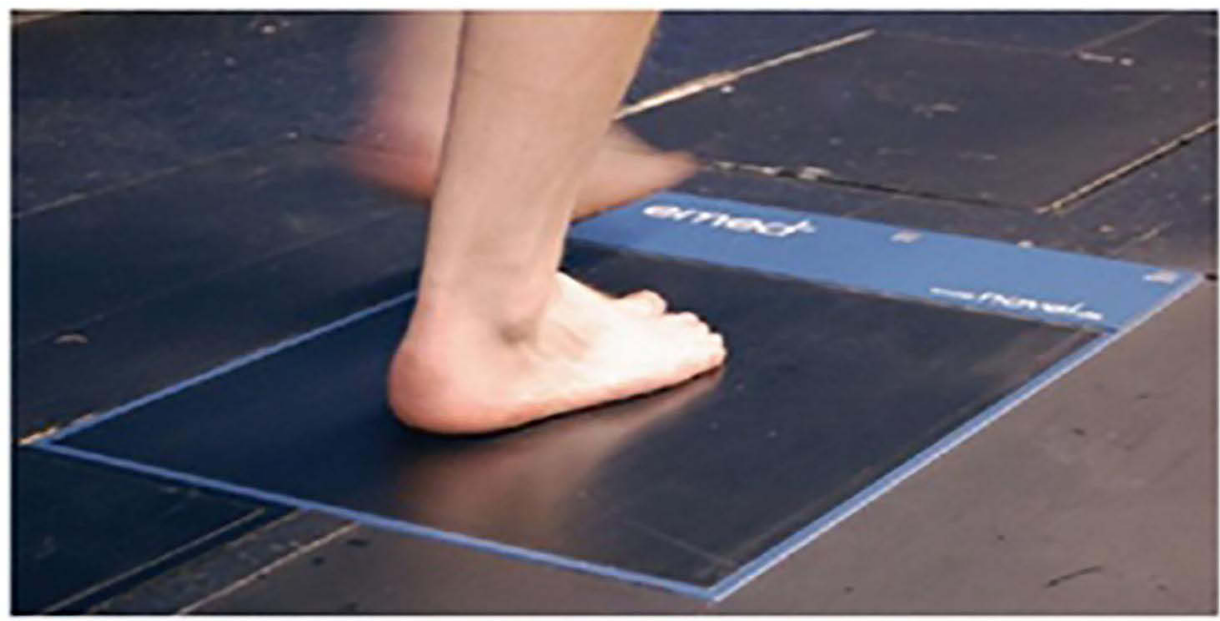

Figure I Plantar pressure platform.

clinical examinations for preserving repeatability and consistency and for avoiding inter-observational differences. The MNSI-q questions were responded by patients, which include common sensory symptoms questions. The system was recommended for comparative differences of plantar pressure distributions under constant calibration and condition before use even though studies indicated that the sensor was sensitive to loading speed, temperature, and surface conditions. The insoles are calibrated to ensure accuracy of measurements.

In different foot areas, peak plantar pressures, plantar pressure at maximum concentration force were recorded in males and females. The subjects walked freely in the department for reproducing their typical gait for approximately 3 minutes prior to executing measurements. Subjects were familiarized with the system when performing several training runs. The study was approved by the institutional review board and was conducted in accordance with the Declaration of Helsinki. The informed consent was taken from the participants prior to enrollment in the study.

The statistical analysis was performed via IBM SPSS statistical software version 25. Kolmogorov-Smirnov test was used to evaluate the data distribution and found normally distributed. Data was described through means and percentages. Independent $t$-test was applied to observe the mean difference in males and females. P- value of $\leq 0.05$ was considered significant.

\section{Results}

Table 1 presents and summarizes the demographic features of study population. The mean age of female and male patients was $50.6 \pm 13.4$ and $46.07 \pm 11.17$, respectively.
The mean difference between the weights of both subjects was $10.43 \pm 3.3$. This shows that male subjects had put more weight as compared to female subjects.

Table 2 presents the bare foot plantar pressure at peak, maximum concentration and maximum force between males and females. The peak plantar pressure between gender in left limb was found significant. Moreover, the mean difference in plantar pressure at maximum concentration and maximum force of right and left limb between males and females was found statistically significant.

Table 3 presents the in-shoe plantar pressure at peak, maximum concentration and maximum force between males and females. The mean difference in plantar pressure at maximum force of left limb between males and females was found statistically significant.

\section{Discussion}

The study was conducted to observe the mean difference in bare foot and in-shoe plantar pressure in diabetic males and females. According to research, 55\% of diabetic ulcers occur on the toes, but $22 \%$ of all ulcers occur in the forefoot and/or mid foot area. ${ }^{31}$ In other investigations, abnormal plantar pressure distribution and increased pressure caused ulcerations, notably at the height of the metatarsophalangeal joints, ${ }^{32}$ and half of the plantar foot ulcers

Table I Demographic Features of Study Population

\begin{tabular}{|l|l|l|}
\hline Variables & Male & Female \\
\hline $\mathrm{n}$ & 30 & 30 \\
Age & $46.07 \pm 11.17$ & $50.6 \pm 13.4$ \\
Weight & $87.21 \pm 18.90$ & $76.78 \pm 15.60$ \\
\hline
\end{tabular}


Table 2 Barefoot Plantar Pressure in Diabetic Males and Females

\begin{tabular}{|l|l|l|l|l|}
\hline Variables & Male & Female & Mean Difference & P-value \\
\hline E-Med peak plantar pressure & & & & \\
\hline Right Limb & $623.67 \pm 330.7 I$ & $636.44 \pm 235.39$ & $12.77 \pm 95.32$ & $>0.05$ \\
\hline Left Limb & $554.17 \pm 265.71$ & $669.77 \pm 221.88$ & $115.6 \pm 43.83$ & $<0.05$ \\
\hline Plantar pressure at Max. Con. Area $\left(\mathrm{cm}^{2}\right)$ & & & & \\
\hline Right Limb & $158.95 \pm 100.75$ & $123.39 \pm 16.34$ & $-35.56 \pm 84.4 I$ & $<0.05$ \\
\hline Left Limb & $157.32 \pm 99.75$ & $123.49 \pm 16.60$ & $-33.83 \pm 83.15$ & $<0.05$ \\
\hline Plantar pressure at Max. Force & & & & \\
\hline Right Limb & $912.66 \pm 363.2$ & $743.66 \pm 200.46$ & $-169.56 \pm 162.74$ & $<0.05$ \\
\hline Left Limb & $908.5 \pm 358.24$ & $765.12 \pm 155.33$ & $-143.83 \pm 202.91$ & $<0.05$ \\
\hline
\end{tabular}

Table 3 In-shoe Plantar Pressure

\begin{tabular}{|l|l|l|l|l|}
\hline Variables & Male & Female & Mean Difference & P-value \\
\hline In-shoe peak plantar pressure & & & & \\
\hline Right Limb & $97.12 \pm 17.65$ & $99.03 \pm 17.87$ & $1.91 \pm 0.22$ & $>0.05$ \\
\hline Left Limb & $194.65 \pm 16.15$ & $197.79 \pm 17.15$ & $3.14 \pm 1$ & $>0.05$ \\
\hline Plantar pressure at Max. Con. Area $\left(\mathrm{cm}^{2}\right)$ & & & & \\
\hline Right Limb & $75.18 \pm 87.55$ & $77.29 \pm 71.14$ & $2.11 \pm 16.41$ & $>0.05$ \\
\hline Left Limb & $75.06 \pm 86.28$ & $73.50 \pm 71.77$ & $-1.56 \pm 14.51$ & $>0.05$ \\
\hline Plantar pressure at Max. Force & & & & \\
\hline Right Limb & $435.027 \pm 495.15$ & $427.60 \pm 437.73$ & $-7.42 \pm 57.42$ & $>0.05$ \\
\hline Left Limb & $474.69 \pm 629.26$ & $456.01 \pm 450.95$ & $18.69 \pm 178.31$ & $<0.05$ \\
\hline
\end{tabular}

were characterized as being situated under the metatarsal heads and hallux. ${ }^{33}$

A study discovered a link between plantar pressure and gender, BMI, and age. In general, the authors found no link between gender and plantar pressure distribution, as they observed in the prior study. ${ }^{34}$ The main difference was that while both males and females had disorders in the forefoot, females had more abnormal plantar pressure in the lateral portion of the foot and mid foot. Hills et $\mathrm{al}^{34}$ also discovered that obese females had higher pressure beneath the mid foot than obese males. In a research, females had higher BMI than men; the authors can only infer that increased body mass causes overload in these locations. However, in our study the barefoot plantar pressure at maximum concentration and force was higher in males than females and in-shoe plantar pressure at maximum force in left limb was also higher in males as in our study the weight of males participants was higher than females.

The overall peak pressures who wore custom-made insoles on the foot soles of the patients were $1.91 \pm 0.22$ for right limb and $3.14 \pm 1$ for left limb. A previous study conducted by Ledoux et $\mathrm{al}^{35}$ to report the overall peak pressures among studied subjects. The study has found in-shoe pressure measurement system and additionally witnessed mean peak pressures of $194 \mathrm{kPa}$ for patients irrespective of ulcers.

Diabetes is witnessed to a pressure imbalance between the rear foot and forefoot throughout walking. Higher peak plantar pressure was shown by Mueller et $\mathrm{al}^{36}$ in diabetic patients in the forefoot since skin deteriorates more often in the forefoot as compared to the rear foot throughout barefoot walking. Anterior 
displacement of weight-bearing was witnessed by Pataky et $\mathrm{al}^{38}$ among diabetic patients. The existence of plantar ulcers was found in forefoot and toe regions. Increased pressure was reported by Zequera et $\mathrm{al}^{38}$ in second toe and metatarsal heads in a small sample size. Additionally, this assessment might be led by neurological modifications such as alternative, motor, and sensorial gait patterns, besides the biomechanical modifications throughout the disease procedure. Similar foot attributes were identified while investigating habitually right and left foot morphology to this study, particularly, wider feet in the toe region, can be reported in barefoot patients.

This study has observed several limitations when explaining the findings from this study. Firstly, subject-specific strike patterns were not taken into consideration specifically for the barefoot population in this study, but this is preliminarily essential for running. In addition, less than $20 \%$ of the barefoot population was a forefoot striker throughout the running test, which is uniform with prior reported percentage of forefoot strikes with self-selected speed.

\section{Conclusion}

By concluding, a pressure shift was witnessed to the forefoot in diabetic patients. As the prevalence of diabetes is increasing, the risk of plantar pressure also increasing simultaneously. The difference in plantar pressure among diabetic males and females is critically important as our study indicated that the bare foot and in-shoe plantar pressure was found higher in males than females as males had higher weight than females. Further longitudinal studies are required to be conducted in this context.

\section{Data Sharing Statement}

The datasets used and analyzed during the current study are available from the corresponding author on reasonable request.

\section{Acknowledgments}

The author is very thankful to all the associated personnel in any reference that contributed in/for the purpose of this research.

\section{Funding}

This research is not funded by any resource.

\section{Disclosure}

The author declares no conflicts of interest in this work.

\section{References}

1. Albert SF, Chen WY. Rigid foot orthoses in the treatment of the neuropathic diabetic foot. Low Extrem. 1996;3:97-106.

2. World Health Organization. 10 Facts About Diabetes. World Health Organization; 2014.

3. International Diabetes Federation (IDF). Diabetes Atlas. Sixth ed. International Diabetes Federation (IDF); 2013.

4. Workbank. Diabetes Prevalence - by Country. MECOMeter. Workbank; 2021.

5. Naeem Z. Burden of diabetes mellitus in Saudi Arabia. Int J Health Sci. 2015;9:V. doi:10.12816/0024690.

6. Altayyar SS. The importance of plantar pressure measurements and appropriate footwear for diabetic patients. J Anal Pharm Res. 2016;3:00057. doi:10.15406/japlr.2016.03.00057

7. Song J, Hillstrom HJ, Neary M, et al. Dynamic barefoot plantar pressure in gait and foot type biomechanics. J Foot Ankle Res. 2014;7:1. doi:10.1186/1757-1146-7-s1-a77

8. Alexiadou K, Doupis J. Management of diabetic foot ulcers. Diabetes Ther. 2012;3:4. doi:10.1007/s13300-012-0004-9

9. Apelqvist J, Bakker K, van Houtum WH, Nabuurs-Franssen MH, Schaper NC. International consensus and practical guidelines on the management and the prevention of the diabetic foot. International Working Group on the Diabetic Foot. Diabetes Metab Res Rev. 2000;16(Suppl 1):S84-S92.

10. Ramanathan AK, Kiran P, Arnold GP, Wang E, Abboud RJ. Repeatability of the pedar-X in-shoe pressure measuring system. Foot Ankle Surg. 2010;16:70-73. doi:10.1016/j.fas.2009.05.006

11. Kim Y, Kim S, Son J, Jeong B. Kinetic role of the metatarsophalangeal joint in normal walking: joint moment and power. Int J Precis Eng Manuf. 2012;13:1481-1485. doi:10.1007/s12541-012-0195-z

12. Cavanagh PR, Simoneau GG, Ulbrecht JS. Ulceration, unsteadiness, and uncertainty: the biomechanical consequence of diabetes mellitus. J Biochech. 1992;26:23-40. doi:10.1016/0021-9290(93)90077-r

13. Boulton AJ, Kirsner RS, Vileikyte L. Clinical practice. Neuropathic diabetic foot ulcers. $N$ Engl J Med. 2004;351:48-55. doi:10.1056/ NEJMcp032966

14. Catanzariti AR, Haverstock BD, Grossman JP, Mendicino RW. Offloading techniques in the treatment of diabetic plantar neuropathic foot ulceration. Adv Wound Care. 1999;12:452-458.

15. Moxey PW, Gogalniceanu P, Hinchliffe RJ, et al. Lower extremity amputations-a review of global variability in incidence. Diabet Med. 2011;28:1144-1153. doi:10.1111/j.1464-5491.2011.03279.x

16. Armstrong DG, Lavery LA, Vela SA, Quebedeaux TL, Fleischli JG. Choosing a practical screening instrument to identify patients at risk for diabetic foot ulceration. Arch Intern Med. 1998;158:289-292. doi:10.1001/archinte.158.3.289

17. LoGerfo FW. Vascular disease, matrix abnormalities, and neuropathy; implications for limb salvage in diabetes mellitus. J Vasc Surg. 1987;5:793-796. doi:10.1016/0741-5214(87)90185-6

18. Payne CB. Biomechanics of the foot in diabetes mellitus: some theoretical considerations. $J$ Am Podiatr Med Assoc. 1998;88:285-289. doi:10.7547/87507315-88-6-285

19. Veves A, Sarnow MR, Giurini JM, et al. Differences in joint mobility and foot pressures between black and white diabetic patients. Diabet Med. 1995;12(7):585-589. doi:10.1111/j.1464-5491.1995.tb00546.x

20. Stess RM, Jensen SR, Mirmiran R. The role of dynamic plantar pressures in diabetic foot ulcers. Diabet Med. 1997;20:855-858. doi:10.2337/diacare.20.5.855

21. Hill MN, Feldman HI, Hilton SC, Holechek MJ, Ylitalo M, Benedict GW. Risk of foot complications in long term diabetic patients with and without ESRD: a preliminary study. Anna J. 1996;23:381-386.

22. Helm PA, Walker SC, Pulliam GF. Recurrence of neuropathic ulceration following healing in a total contact cast. Arch Phys Med Rehabil. 1991;72:967-970. 
23. Moss SE, Klein R, Klein BEK. The prevalence and incidence of lower extremity amputation in a diabetic population. Arch Intern Med. 1992;152:610-616. doi:10.1001/archinte.152.3.610

24. Tafti N, Turk SB, Hemmati F, et al. Effect of wearing insole with different density on standing and walking plantar pressure distribution. J Mech Med Bio. 2019;19(03):1950006. doi:10.1142/ S0219519419500064

25. Tsung BY, Zhang M, Mak AF, Wong MW. Effectiveness of insoles on plantar pressure redistribution. $J$ Rehab Res Develop. 2004;41:767. doi:10.1682/JRRD.2003.09.0139

26. Ahmed S, Barwick A, Butterworth P, Nancarrow S. Footwear and insole design features that reduce neuropathic plantar forefoot ulcer risk in people with diabetes: a systematic literature review. $J$ Foot Ankle Res. 2020;13:1-3. doi:10.1186/s13047-020-00400-4

27. Tang UH, Zügner R, Lisovskaja V, Karlsson J, Hagberg K, Tranberg R. Comparison of plantar pressure in three types of insole given to patients with diabetes at risk of developing foot ulcers-a two-year, randomized trial. J Clin Trans Endocrinol. 2014;1 (4):121-132.

28. Mickle KJ, Cliff DP, Munro BJ, Okely AD, Steele JR. Relationship between plantar pressures, physical activity and sedentariness among preschool children. J Sci Med Sport. 2011;14:36-41. doi:10.1016/j. jsams.2010.05.005

29. Wei Z, Zhang Z, Jiang J, Zhang Y, Wang L. Comparison of plantar loads among runners with different strike patterns. J Sports Sci. 2019;37:2152-2158. doi:10.1080/02640414.2019.1623990

30. Mickle KJ, Steele JR. Obese older adults suffer foot pain and foot-related functional limitation. Gait Posture. 2015;42 (4):442-447. doi:10.1016/j.gaitpost.2015.07.013

31. Prompers L, Huijberts M, Apelqvist J. High prevalence of ischaemia, infection and serious comorbidity in patients with diabetic foot disease in Europe. Baseline results from the Eurodiale study. Diabetologia. 2007;50(1):18-25. doi:10.1007/s00125-006-0491-1
32. Bowling FL, Reeves ND, Boulton AJ. Gait-related strategies for the prevention of plantar ulcer development in the high risk foot. Curr Diabetes Rev. 2011;7(3):159-163. doi:10.2174/157339911795843159

33. Dinh TL, Veves A. A review of the mechanisms implicated in the pathogenesis of the diabetic foot. Int J Low Extrem Wounds. 2005;4 (3):154-159. doi:10.1177/1534734605280130

34. Hills AP, Hennig EM, McDonald M, Bar-Or O. Plantar pressure differences between obese and non-obese adults: a biomechanical analysis. Int J Obes Relat Metabol Disord. 2001;25(11):1674-1679. doi:10.1038/sj.ijo.0801785

35. Ledoux WR, Shofer JB, Cowley MS, Ahroni JH, Cohen V, Boyko EJ. Diabetic foot ulcer incidence in relation to plantar pressure magnitude and measurement location. $J$ Diab Compl. 2013;27:621-626. doi:10.1016/j.jdiacomp.2013.07.004

36. Mueller MJ, Hastings M, Commean PK, et al. Forefoot structural predictors of plantar pressures during walking in people with diabetes and peripheral neuropathy. $J$ Biomech. 2003;36:1009-1017. doi:10.1016/S0021-9290(03)00078-2

37. Pataky TC, Caravaggi P, Savage R, et al. New insights into the plantar pressure correlates of walking speed using pedobarographic statistical parametric mapping (pSPM). $J$ Biomech. 2008;41:1987-1994. doi:10.1016/j.jbiomech.2008.03.034

38. Zequera M, Stephan S, Paul J Effectiveness of moulded insoles in reducing plantar pressure in diabetic patients. In 2007 29th Annual International Conference of the IEEE Engineering in Medicine and Biology Society; 2007; 4671-4674. IEEE.
Medical Devices: Evidence and Research

\section{Publish your work in this journal}

Medical Devices: Evidence and Research is an international, peerreviewed, open access journal that focuses on the evidence, technology, research, and expert opinion supporting the use and application of medical devices in the diagnosis, monitoring, treatment and management of clinical conditions and physiological processes. The identification of novel devices and optimal use of existing devices which will lead to improved clinical outcomes and more effective patient management and safety is a key feature of the journal. The manuscript management system is completely online and includes a very quick and fair peer-review system. Visit http:// www.dovepress.com/testimonials.php to read real quotes from published authors. 